\title{
Hacia una comunicación corporativa transmedia
}

\author{
Carlos Molina \\ Director general de Best Relations
}

\section{Referencia de este artículo}

Molina, Carlos (2017). Hacia una comunicación corporativa transmedia. En: adComunica. Revista Científica de Estrategias, Tendencias e Innovación en Comunicación, $\mathrm{n}^{0} 14$. Castellón: Asociación para el Desarrollo de la Comunicación adComunica y Universitat Jaume I, 259-265. DOI: http://dx.doi. org/10.6035/2174-0992.2017.14.15

\section{Introducción}

Cuando pensamos en el desarrollo y aplicación de narrativas transmedia en el mundo de las marcas, solemos hacerlo vinculándolas a la comunicación de producto o servicio. Lo transmediático se convierte así en un instrumento de la relación entre empresa y consumidor o cliente. Éste es el sujeto del contenido, al que se dirigen los mensajes y al que se le da la capacidad de profundizar hasta donde desee en la historia que se le plantea. En sus manos queda la posibilidad de hacerla crecer, de modificarla, de transformarla hasta hacerla suya. Como viene a decir el profesor Joan Costa, la marca es una construcción social (Costa, 2012) y, por lo tanto, la propiedad emocional pertenece a los públicos que se relacionan con ella. 
El razonamiento anterior, sin embargo, deja a un lado la comunicación corporativa y, de forma más específica, la comunicación interna. El motivo suele estar en considerar que la relación entre marcas y personas sólo puede tener lugar en un marco comercial. En ese escenario, la comunicación digital ha eliminado intermediarios y ha acercado los extremos, simplificando el lenguaje, generando experiencias más próximas y personalizadas, y provocando un nivel de emoción entre empresas y consumidores desconocido hasta hace sólo una década.

El caso de Nestlé contra Greenpeace marcó un punto de inflexión. En 2010, una campaña de la ONG contra la multinacional por el uso de aceite de palma en la fabricación de las chocolatinas Kit Kat derivó en una llamada a los internautas para modificar el logotipo de la compañía como parte de los actos de denuncia. La reacción de Nestlé, avisando de que actuaría eliminando cualquier contenido en su página de Facebook que supusiera la modificación de su marca, provocó un "efecto Streissand" (Colado, 2017) que amplificó la notoriedad de la acción. La presión de los consumidores y el impacto en las redes sociales llevó a Nestlé a dar explicaciones, pedir disculpas y anunciar que dejaría de utilizar productos que supusieran la deforestación de los bosques en los que habitan los orangutanes de Indonesia (Greenpeace, 2010), objeto de la defensa iniciada por Greenpeace.

Desde entonces, la consciencia de que los usuarios no sólo tienen la capacidad de presionar, sino de que son agentes activos en la construcción de las marcas, se aprovechó de forma directa en campañas de publicidad y en acciones de marketing. Nutella, por ejemplo, no dudó en dejar en manos de los clientes su logotipo para dejarles crear, a través de Facebook, etiquetas personalizadas que podrían adherir a botes de su crema de chocolate (Marketing News, 2013). Coca-Cola, poco después, inició una acción similar cambiando en las latas de su refresco el icónico logo por nombres de personas (Jay, 2014). Por primera vez, se estaba invitando a los clientes a hacer suyas las marcas, transformándolas tal y como las sintieran.

El beneficio de este comportamiento para las organizaciones estaba claro: mayor empatía con los públicos, incremento del alcance de las publicaciones relacionadas con las acciones, aumento del engagement (entendido como la relación entre el alcance de una publicación y las reacciones generadas por la misma) y giro del valor de los contenidos hacia la positividad de marca.

En un contexto como éste cobran sentido las narrativas transmedia. Es cierto que no necesitaban de las redes sociales para ser una realidad porque, de hecho, tenemos ejemplos históricos que cumplen con buena parte de sus características. En las últimas décadas, el universo Star Wars o más recientemente el mundo de la serie "Breaking Bad" ejemplifican como pocos lo que representa este concepto. 


\section{Transmedia y organizaciones}

Que en los últimos años apenas se haya hablado de transmedia en relación con los entornos corporativos y la comunicación interna tiene que ver con la percepción que se ha tenido, de puertas adentro en las organizaciones, del papel de las marcas y las comunidades propias. ¿Tienen más relevancia los grupos de consumidores digitalizados que los de empleados conectados a los medios sociales sólo porque el alcance de los primeros es potencialmente mayor y están más asociados a los procesos de ventas? No necesariamente.

Para entender por qué no, es importante revisar las cinco fases en las que se ha movido la comunicación interna digital en las empresas en la última década. En muchas de ellas, las fases se superponen. A menudo podemos observar que la velocidad a la que se suceden las etapas es desigual, dependiendo del grado de madurez o del contexto corporativo de cada institución.

Fase 1: Prohibición. La organización considera un problema de seguridad y de productividad el acceso a entornos sociales desde el puesto de trabajo. Para ello, establece barreras técnicas a la navegación.

Fase 2: Regulación. Los empleados son seres sociales y, al margen de la organización, tienen presencia en las redes con sus propios perfiles. Además, las barreras técnicas pierden sentido una vez que se generaliza el acceso a internet desde teléfonos móviles. En este punto, las compañías establecen marcos de regulación internos para informar a sus profesionales de los límites de la comunicación digital en relación con la propia empresa. Son las llamadas social media policies, o políticas de uso de los medios sociales, y definen las implicaciones que puede tener compartir información corporativa con cierto detalle o cierto tono.

Fase 3: Identificación. A medida que se asume el carácter social de las personas en la organización, el seguimiento de su actividad descubre su capacidad de liderazgo en ciertos ámbitos de conversación. Comienza la identificación de embajadores de marca internos.

Fase 4: Advocacy. Más allá de los líderes, todos los empleados tienen la capacidad de impulsar los mensajes de la organización en los medios sociales. Actúan como micro-influencers, es decir, influyentes de nicho que ayudan a la dispersión de líneas narrativas y argumentales entre grupos más específicos. En algunos casos, las empresas aprovechan ese potencial para sugerirles contenidos para compartir a través de sus perfiles, de forma que configuren una marca personal más potente y, además, expresen su adhesión a la empresa de manera pública. Esto puede hacerse a través de plataformas específicas, como LinkedIn Elevate o SocialReacher.

Fase 5: Ingagement. Concepto desarrollado desde Best Relations (Molina, 2017) que alude a un estado de afinidad entre públicos internos y organización que permite la construcción y difusión conjunta de valores y mensajes corporativos 
de forma orgánica, sin anteponer normas internas porque prima la responsabilidad y la confianza que se le supone a cada profesional. La relación entre las personas se canaliza a través de la cooperación que proporcionan las herramientas sociales.

Esta evolución implica que es muy difícil en las dos primeras fases de la comunicación digital interna aplicar narrativas transmedia, puesto que se carece de confianza en el interlocutor y, por lo tanto, no hay un escenario propicio para dejar en sus manos la construcción social de la marca. A partir de la fase 3, cambian los condicionantes y a medida que avanzamos hacia el estadio del “ingagement”, se generan más oportunidades para construir un relato con los empleados en el que sean parte activa de la conversación.

\section{Aplicación corporativa del discurso transmedia}

Desde este punto de vista y con esta perspectiva de evolución, cobran sentido con matices particulares las características de las narrativas transmedia, tal y como las enumera Diego Rivera, director de Estrategia y Creatividad de Best Relations (Rivera, 2017).

Narrativa. Los objetivos de las empresas pueden articularse en torno a hilos narrativos (planteamiento, nudo, desenlace). No tienen sentido sólo para los públicos externos, sino también para los internos, para quienes es importante ser los primeros que conocen los propósitos de la empresa y poder participar de los mismos. Una organización que sabe hacia dónde quiere ir puede plantear acciones con sentido en las que implicar a sus empleados. Maersk, por ejemplo, articula su narrativa en torno al transporte marítimo, y sus empleados más expertos aportan contenido desde los blogs especializados de la compañía, añadiendo “capítulos” a ese storytelling.

Desdoblamiento de canales. El discurso corporativo puede expandirse en diferentes plataformas digitales, dando sentido así a una comunicación orientada a públicos distintos. La sala de prensa digital se dirige a los medios de comunicación, las redes sociales públicas a los públicos externos, y las redes sociales internas y plataformas de colaboración, a los públicos internos (empleados, socios y proveedores). En cada espacio, las experiencias son diferentes.

Profundidad. El interés por un tipo de contenidos u otro se puede traducir en el uso de diferentes formatos que den respuesta a distintas necesidades de información. Los resultados anuales de la compañía pueden detallarse con precisión en la memoria anual -accesible desde la web corporativa-, pero también pueden resumirse en vídeo o en infografías.

Generación de experiencias. Es uno de los aspectos más importantes de la narrativa transmedia, y cobra mucho sentido cuando se avanza hacia un contexto en el que el empleado necesita sentirse apoyado por la organización y orgulloso 
de pertenecer a ella. Personalizar, por ejemplo, un kit de bienvenida cuando un profesional se incorpora a la empresa, o celebrar iniciativas de cooperación para el desarrollo de proyectos innovadores (intraemprendimiento) fortalece lazos y genera experiencias únicas que incrementan el vínculo entre compañía y personas.

Autonomía del usuario. Los entornos digitales incrementan la posibilidad de elegir vías alternativas para hacer las cosas. En los entornos corporativos, el uso de plataformas de cooperación y trabajo en la nube están incidiendo en ello $\mathrm{y}$, por lo tanto, en la manera en que se articula el discurso. Las herramientas de organización del trabajo (Trello, 365, Basecamp), las plataformas de gestión de grupos (Slack, Grupos de Google) o de comunicación multimedia (Hangouts, Skype, Adobe Connect) ofrecen formas diferentes de acceder a la información, construir historias con los compañeros e incluso prescindir de unas herramientas en favor de otras. ¿Tiene sentido el correo electrónico como vía de comunicación eficiente frente a plataformas colaborativas?

Gamificación. El uso de las dinámicas de juegos ayuda a aprender y recordar mejor. Algunas empresas usan estas técnicas para reconocer las aportaciones de valor de los miembros de la organización (por ejemplo, mediante insignias en una red social interna), destacarlos para su identificación por el resto del grupo y, al mismo tiempo, incentivar los procesos de participación y descubrimiento de nuevos contenidos.

Uso de segundas y terceras pantallas. Cada vez es más frecuente en los entornos corporativos apoyarse en el uso de aplicaciones móviles para acercar la información de utilidad para el emplado a entornos dinámicos. No es lo mismo gestionar la participación en grupos desde el ordenador que hacerlo en movilidad, aprovechando los micromomentos disponibles. Además, las segundas y terceras pantallas (por ejemplo, circuitos cerrados de televisión en una oficina) pueden aportar datos o información complementaria a la que ya se recibe por otro tipo de canales (boletines por correo electrónico, revistas corporativas, webs, etc.).

Generación de emociones. Sin duda, es uno de los elementos más importantes en la narrativa transmedia. La clave reside en la capacidad para convertir la información corporativa en algo cercano a los problemas y necesidades de los miembros de la organización, y en poner nombre y apellidos, personalizando la narrativa. Cuando la empresa no habla de productos, sino de personas, y pone por delante a sus expertos como portavoces, humaniza su comunicación, empatiza con públicos internos y externos, y se pone al nivel de sus clientes.

La narrativa transmedia en entornos corporativos exige, a la postre, una coherencia traducida en la generación de entornos con cultura social. Esto implica que debe tratarse con el mismo interés y atención a los públicos internos que a los externos. Y ello supone que tengamos presente que no podemos exigir elevados niveles de participación activa a todos, sino niveles de participación sinceros. De hecho, cada vez están más presentes en los espacios digitales los que, 
desde Best Relations, calificamos como obsumers (Cordero, 2016), un híbrido entre observers y consumers, variación del prosumer del que hablaba Alvin Toffler (Kotler, 1986). Son usuarios que consumen el contenido, pero son absolutamente pasivos no ya en su generación, sino en la interacción con el mismo.

En definitiva, la comunicación corporativa sí puede ser transmedia, aprovechando sus diferentes elementos para potenciar entornos más colaborativos, humanos y productivos que los que definen los entornos empresariales convencionales. Antes, eso sí, será necesario incorporar nuevas rutinas sociales. Cada cual tendrá que encontrar sus necesidades y su ritmo de cambio.

\section{Referencias bibliográficas}

Costa, Juan (2012). Construcción y gestión estratégica de la marca: Modelo MasterBrand. En: Revista Luciérnaga, ${ }^{0} 4$, 8. Medellín, Colombia: Grupo de Investigación en Comunicación, Facultad de Comunicación Audiovisual, Politécnico Colombiano Jaime Isaza Cadavid, 20-25.

Kotler, Philip. (1986). The Prosumer Movement : a New Challenge For Marketers. En: NA - Advances in Consumer Research, $\mathrm{n}^{0} 13$, Richard J. Lutz, Provo, UT: Association for Consumer Research, 510-513.

\section{Webgrafía}

Colado, Pablo. ¿Qué es el efecto streissand? Muy Interesante [consultado en 2017-03-19]. Disponible en http://www.muyinteresante.es/curiosidades/preguntas-respuestas/que-es-el-efecto-streisand-951390994451

Cordero, Alicia (2016). Este muerto está muy vivo o cómo el perfil de usuarios de redes sociales está cambiando. Medios Sociales, 21/12/2016 [Consultado 19/03/2017]. Disponible en http://mediossociales.es/usuarios-de-redes-socialescambiando/

La mala gestión de comunicación online en el “caso Nestlé”. Tu Escaparate Virtual, 23/05/2012 [Consultado 19/03/2017]. Disponible en https://tuescaparatevirtual.wordpress.com/2012/05/23/caso-nestle/

Molina, Carlos (2017). Ingagement: la afinidad de la comunicación interna. Medios Sociales, 9/03/2017 [Consultado 19/03/2017]. Disponible en http://mediossociales.es/ingagement-comunicacion-interna/

Moye, Jay (2014). Comparte una Coca-cola: Cómo la innovadora campaña empezó en Australia y Nueva Zelanda. Coca-Cola Journey, 25/09/2014 [Consultado 19/03/2017]. Disponible en http://journey.coca-cola.com/historias/comparte-unacoca-cola-como-la-innovadora-campana-empezo-en-australia-y-nueva-zelanda 
Nutella amplía su promoción de etiquetas personalizadas por su gran éxito. Marketing News, 11/09/2013 [Consultado 19/03/2017]. Disponible en http:// www.marketingnews.es/gran-consumo/noticia/1076799028005/nutella-ampliapromocion-etiquetas.1.html

Rivera, Diego (2012). Transmedia y storytelling: casos y ejemplos. Medios Sociales, 02/11/2012 [Consultado 19/03/2017]. Disponible en http://mediossociales.es/transmedia-storytelling-ejemplos/

Sweet success for Kit Kat campaign: you asked, Nestlé has answered. Greenpeace, 17/05/2010 [Consultado 19/03/2017]. Disponible en http://www.greenpeace.org/international/en/news/features/Sweet-success-for-Kit-Kat-campaign/ 